\title{
Supporting Information: Stability of the Double Gyroid Phase in Bottlebrush Diblock Copolymer Melts
}

\author{
So Jung Park, Guo Kang Cheong, Frank S. Bates, and Kevin D. Dorfman* \\ Department of Chemical Engineering and Materials Science, University of Minnesota- \\ Twin Cities, 421 Washington Ave. SE, Minneapolis, MN 55455, United States \\ E-mail: dorfman@umn.edu
}

\section{A SCFT formalism for bottlebrush block polymers and simulation details}

We consider an incompressible polymer melt consisting of $n$ identical bottlebrush diblock polymers in a volume $V$, where each monomer has the same statistical segment length $b=b_{\mathrm{A}}=b_{\mathrm{B}}$. As illustrated in Figure $\mathrm{S} 1$, the $\mathrm{AB}$ diblock backbone of statistical segment number $N_{\mathrm{BB}}$ has $n_{\mathrm{SC}, \mathrm{A}} \mathrm{A}$ grafts and $n_{\mathrm{SC}, \mathrm{B}} \mathrm{B}$ grafts of identical length $N_{\mathrm{SC}}$. The total number of grafts, $n_{\mathrm{SC}}=n_{\mathrm{SC}, \mathrm{A}}+n_{\mathrm{SC}, \mathrm{B}}$, are evenly spaced by $d=N_{\mathrm{BB}} / n_{\mathrm{SC}}$, starting with the first side chain grafted $d / 2$ from the A backbone end. The backbone contour is parameterized starting from $s=0$ at the $\mathrm{A}$ end and ending at $s=N_{\mathrm{BB}}$ at the $\mathrm{B}$ end. The backbone can be envisioned as containing $n_{\mathrm{SC}}+1$ segments based on the graft points; the AB junction of the backbone is defined as the midpoint of the backbone segment which is divided by one A and one B side chain to avoid ambiguity of defining the type of grafts when the junction is at grafting points, so that the $\mathrm{AB}$ junction is located at $s^{*} \equiv N_{\mathrm{BB}} f_{\mathrm{A}}$ where the volume 


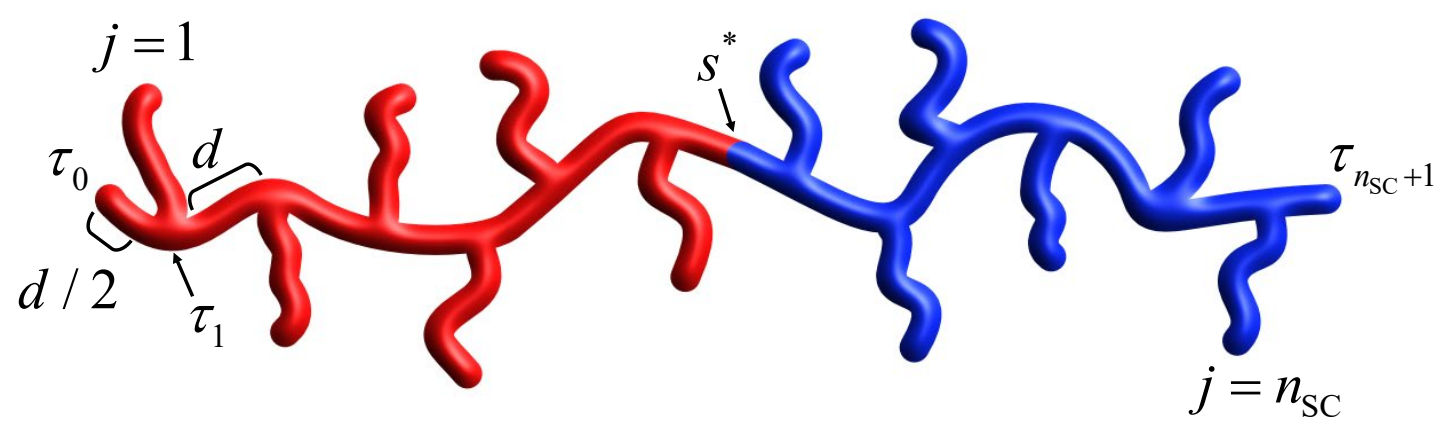

Figure S1: Schematic of the AB bottlebrush block polymer model.

fraction $f_{\mathrm{A}}$ is equal to $n_{\mathrm{SC}, \mathrm{A}} / n_{\mathrm{SC}}$ in this model.

The forward propagator for a side chain of type $\alpha=\mathrm{A}$ or B starting from a free end is evaluated from the solution of the modified diffusion equation

$$
\frac{\partial}{\partial s} q_{\mathrm{SC}, \alpha}(\mathbf{r}, s)=\left[\frac{b^{2}}{6} \nabla^{2}-\omega_{\alpha}(\mathbf{r})\right] q_{\mathrm{SC}, \alpha}(\mathbf{r}, s)
$$

with an initial condition $q_{\mathrm{SC}, \alpha}(\mathbf{r}, 0)=1$, and the parameter $s$ ranges from 0 to $N_{\mathrm{SC}}$. Here, $\omega_{\alpha}(\mathbf{r})$ is the effective chemical potential field acting on monomer of type $\alpha$ at position $\mathbf{r}$, and it will be self-consistently determined by the mean field equations, which will be explained later.

The forward propagator for a backbone starting from the A end is obtained from the $i^{\text {th }}$ divided backbone segment,

$$
q_{\mathrm{BB}}(\mathbf{r}, s)=q_{\mathrm{BB}}^{(i)}(\mathbf{r}, s), \quad \tau_{i} \leq s<\tau_{i+1} \quad i=0,1,2, \ldots, n_{\mathrm{SC}}
$$

where

$$
\tau_{i}=\frac{d}{2}+(i-1) d \quad i=1,2, \ldots, n_{\mathrm{SC}}
$$

specifies each grafting point, and $\tau_{0}=0$ and $\tau_{n_{\mathrm{SC}}+1}=N_{\mathrm{BB}}$ for the two free backbone ends. 
The propagators satisfy

$$
\frac{\partial}{\partial s} q_{\mathrm{BB}}^{(i)}(\mathbf{r}, s)=\left[\frac{b^{2}}{6} \nabla^{2}-\omega_{\alpha(s)}(\mathbf{r})\right] q_{\mathrm{BB}}^{(i)}(\mathbf{r}, s)
$$

with initial conditions

$$
\begin{aligned}
q_{\mathrm{BB}}^{(0)}(\mathbf{r}, 0) & =1 \\
q_{\mathrm{BB}}^{(i)}\left(\mathbf{r}, \tau_{i}\right) & =q_{\mathrm{BB}}^{(i-1)}\left(\mathbf{r}, \tau_{i}\right) q_{\mathrm{SC}, \alpha\left(\tau_{i}\right)}\left(\mathbf{r}, N_{\mathrm{SC}}\right), \quad i=1,2, \ldots, n_{\mathrm{SC}}
\end{aligned}
$$

where $\alpha(s)$ switches from A to B at $s=s^{*}$.

The backward propagator for a backbone starting from the other end is also obtained from the $i^{\text {th }}$ backbone segment,

$$
q_{\mathrm{BB}}^{\dagger}(\mathbf{r}, s)=q_{\mathrm{BB}}^{\dagger(i)}(\mathbf{r}, s), \quad \tau_{i} \leq s<\tau_{i+1} \quad i=0,1,2, \ldots, n_{\mathrm{SC}}
$$

which satisfies the following modified diffusion equation

$$
-\frac{\partial}{\partial s} q_{\mathrm{BB}}^{\dagger(i)}(\mathbf{r}, s)=\left[\frac{b^{2}}{6} \nabla^{2}-\omega_{\alpha(s)}(\mathbf{r})\right] q_{\mathrm{BB}}^{\dagger(i)}(\mathbf{r}, s)
$$

with initial conditions

$$
\begin{aligned}
& q_{\mathrm{BB}}^{\dagger\left(n_{\mathrm{SC}}\right)}\left(\mathbf{r}, N_{\mathrm{BB}}\right)=1 \\
& q_{\mathrm{BB}}^{\dagger(i-1)}\left(\mathbf{r}, \tau_{i}\right)=q_{\mathrm{BB}}^{\dagger(i)}\left(\mathbf{r}, \tau_{i}\right) q_{\mathrm{SC}, \alpha\left(\tau_{i}\right)}\left(\mathbf{r}, N_{\mathrm{SC}}\right), \quad i=1,2, \ldots, n_{\mathrm{SC}}
\end{aligned}
$$

Lastly, the backward propagator $q_{\mathrm{SC}, \alpha}^{\dagger(j)}(\mathbf{r}, s)$ for $j^{\text {th }}$ side chain of type $\alpha$ starting from a grafting point is calculated by solving

$$
-\frac{\partial}{\partial s} q_{\mathrm{SC}, \alpha}^{\dagger(j)}(\mathbf{r}, s)=\left[\frac{b^{2}}{6} \nabla^{2}-\omega_{\alpha}(\mathbf{r})\right] q_{\mathrm{SC}, \alpha}^{\dagger(j)}(\mathbf{r}, s), \quad j=1,2, \ldots, n_{\mathrm{SC}}
$$


with initial conditions

$$
q_{\mathrm{SC}, \alpha}^{\dagger(j)}\left(\mathbf{r}, N_{\mathrm{SC}}\right)=q_{\mathrm{BB}}^{(j-1)}\left(\mathbf{r}, \tau_{j}\right) q_{\mathrm{BB}}^{\dagger(j)}\left(\mathbf{r}, \tau_{j}\right), \quad j=1,2, \ldots, n_{\mathrm{SC}}
$$

Using all propagator quantities obtained by solving eqns A1, A4, A8, and A11, the A and B segment densities in units of inverse monomer volume are determined by

$$
\begin{aligned}
& \phi_{\mathrm{A}}(\mathbf{r})=\frac{1}{Q N_{\mathrm{tot}}}\left(\int_{0}^{N_{\mathrm{SC}}} d s q_{\mathrm{SC}, \mathrm{A}}(\mathbf{r}, s) \bar{q}_{\mathrm{SC}, \mathrm{A}}^{\dagger}(\mathbf{r}, s)+\int_{0}^{s^{*}} d s q_{\mathrm{BB}}(\mathbf{r}, s) q_{\mathrm{BB}}^{\dagger}(\mathbf{r}, s)\right) \\
& \phi_{\mathrm{B}}(\mathbf{r})=\frac{1}{Q N_{\mathrm{tot}}}\left(\int_{0}^{N_{\mathrm{SC}}} d s q_{\mathrm{SC}, \mathrm{B}}(\mathbf{r}, s) \bar{q}_{\mathrm{SC}, \mathrm{B}}^{\dagger}(\mathbf{r}, s)+\int_{s^{*}}^{N_{\mathrm{BB}}} d s q_{\mathrm{BB}}(\mathbf{r}, s) q_{\mathrm{BB}}^{\dagger}(\mathbf{r}, s)\right) \\
& \bar{q}_{\mathrm{SC}, \alpha}^{\dagger}(\mathbf{r}, s) \equiv \sum_{j \in \alpha}^{n_{\mathrm{SC}}} q_{\mathrm{SC}, \alpha}^{\dagger(j)}(\mathbf{r}, s), \quad \alpha=\mathrm{A} \text { or B }
\end{aligned}
$$

where $Q$ is the total partition function of the molecule,

$$
Q=\frac{1}{V} \int d \mathbf{r} q_{\mathrm{BB}}\left(\mathbf{r}, N_{\mathrm{BB}}\right)
$$

and $N_{\text {tot }}=N_{\mathrm{BB}}+n_{\mathrm{SC}} N_{\mathrm{SC}}$ is total number of statistical segments in a molecule. In the mean field approximation, the densities should self-consistently satisfy the following mean field equations:

$$
\begin{aligned}
& \omega_{\mathrm{A}}(\mathbf{r})=\chi \phi_{\mathrm{B}}(\mathbf{r})+\xi(\mathbf{r}) \\
& \omega_{\mathrm{B}}(\mathbf{r})=\chi \phi_{\mathrm{A}}(\mathbf{r})+\xi(\mathbf{r})
\end{aligned}
$$

where $\chi$ is the Flory-Huggins interaction parameter and $\xi(\mathbf{r})$ is the pressure field enforcing the incompressiblity constraint, $\phi_{\mathrm{A}}(\mathbf{r})+\phi_{\mathrm{B}}(\mathbf{r})=1$. After iteratively solving those self-consistent quantities, the Helmholtz free energy per chain can be calculated as

$$
\frac{F}{n k_{\mathrm{B}} T}=-\ln (e Q)-\frac{N_{\mathrm{tot}}}{V} \int d \mathbf{r}\left(\omega_{\mathrm{A}}(\mathbf{r}) \phi_{\mathrm{A}}(\mathbf{r})+\omega_{\mathrm{B}}(\mathbf{r}) \phi_{\mathrm{B}}(\mathbf{r})\right)+\frac{\chi N_{\text {tot }}}{V} \int d \mathbf{r} \phi_{\mathrm{A}}(\mathbf{r}) \phi_{\mathrm{B}}(\mathbf{r})
$$


In the lamellar morphology, the positional probability distributions of each segment in the $j^{\text {th }}$ grafted $\gamma$ type side chain can be calculated by

$$
\begin{aligned}
P_{\mathrm{SC}, \gamma}^{(j)}(z, s) & \equiv \frac{q_{\mathrm{SC}, \gamma}(z, s) q_{\mathrm{SC}, \gamma}^{\dagger(j)}(z, s)}{Q} \\
Q & =\frac{1}{L} \int d z q_{\mathrm{BB}}\left(z, N_{\mathrm{BB}}\right)
\end{aligned}
$$

where $s=0$ at the free ends and $s=N_{\mathrm{SC}}$ at the grafting points. The distribution function $P_{\mathrm{SC}, \gamma}^{(j)}(z, s)$ is normalized such that the spatial integral over one unit cell is the lamellar period $L$. The density profile of $\gamma$-type side chains $\phi_{\mathrm{SC}, \gamma}$ and that of $\gamma$-type backbone $\phi_{\mathrm{BB}, \gamma}$ in Figure 3 of the main text are obtained by calculating the first and second terms in the parenthesis in eqn A13 or A14, respectively.

The above SCFT formalism can be readily converted to the formalism for coil-bottlebrush polymer models we used in this work by modifying the relative grafting positions $\tau_{i}$ 's in eqn A3 and $\mathrm{AB}$ junction position $s^{*}$ as we modeled coil(A)-bottlebrush(B) polymer by taking all of the A type side chains of the $\mathrm{AB}$ bottlebrush block polymer and putting them at the end of the A backbone block. The corresponding bottlebrush(A)-coil(B) polymer was constructed by the same procedure, this time moving the B-type side chains to the end of the B backbone block.

We implemented the pseudo-spectral method of improved accuracy, as described by Ranjan et al., ${ }^{1}$ for solving SCFT diffusion equations A1, A4, A8, and A11 with periodic boundary conditions. We also used the Anderson-mixing scheme with stress relaxation ${ }^{2}$ for updating the $\omega$ fields. In order to accelerate the computations, we adopted the method first suggested by Levi et al. ${ }^{3}$ for solving $\bar{q}_{\mathrm{SC}, \alpha}^{\dagger}(\mathbf{r}, s)$ in eqn A15. Since the modified diffusion equation A11 is linear, $\bar{q}_{\mathrm{SC}, \alpha}^{\dagger}(\mathbf{r}, s)$ also satisfies that equation. Therefore, instead of solving for each $q_{\mathrm{SC}, \alpha}^{\dagger(j)}$ separately, we solve the modified diffusion equation once for each chemical type with summed initial conditions, eqn A12. In this way, the computational time does not increase linearly with the total number of side chains, and the computation for highly grafted bottlebrush 
polymers with $n_{\mathrm{SC}}=200$ is available within a few hours. The SCFT calculations used integration steps of $0.0001 \leq \Delta s \leq 0.0025$ and spatial grid sizes of $52^{d}$ for coil-bottlebrush polymer and $40^{d}$ for the others, where $d$ is the dimension of system $(d=3$ for spherical and double gyroid phases, $d=2$ for cylindrical phases, and $d=1$ for lamellar phases).

\section{B Phase diagrams for bottlebrush block polymer melts}
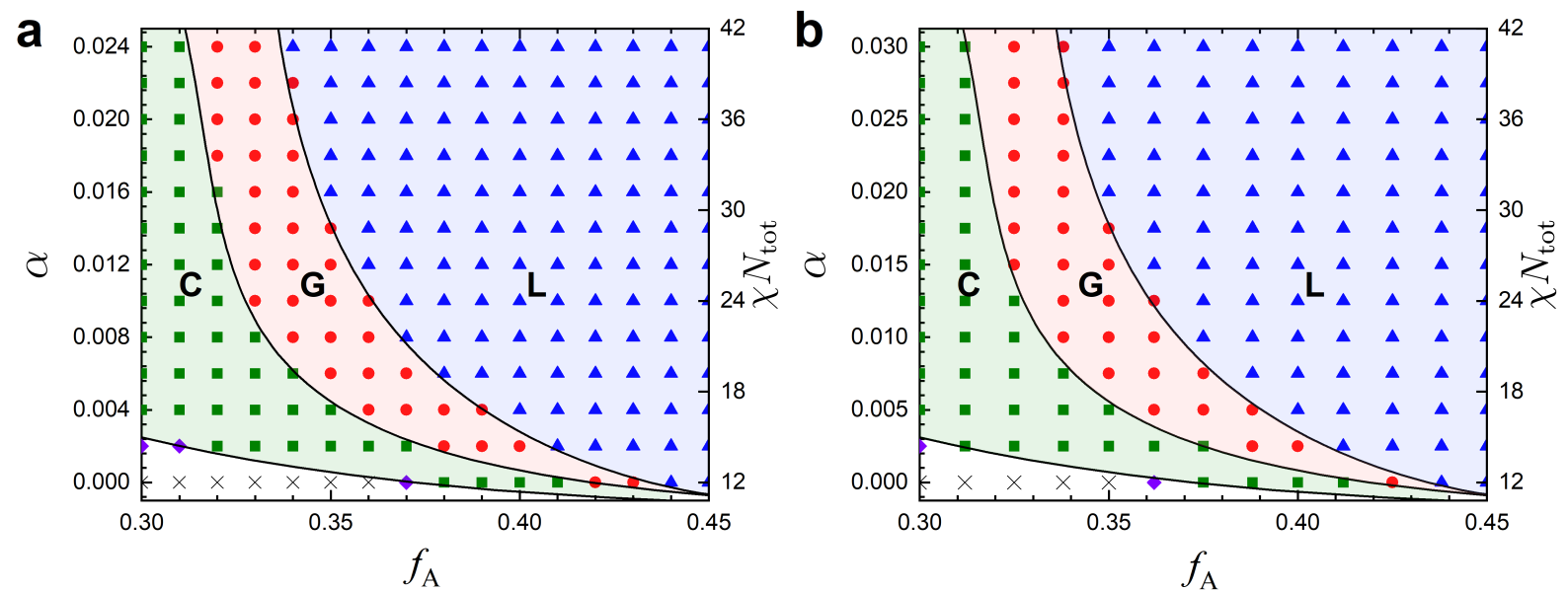

Figure S2: Phase diagrams of crewcut bottlebrush block polymers of (a) $n_{\mathrm{SC}}=100$ and (b) $n_{\mathrm{SC}}=80$ at $\chi=12$. The black lines are phase boundaries of diblock copolymers at equivalent $\chi N=\chi N_{\text {tot }}$. The stability regions of lamellar (L), hexagonal cylindrical (C), and double gyroid $(\mathrm{G})$ phases are labeled, and the purple diamond and black cross-mark symbols denote bcc spherical phase and homogeneous disordered phase, respectively. 


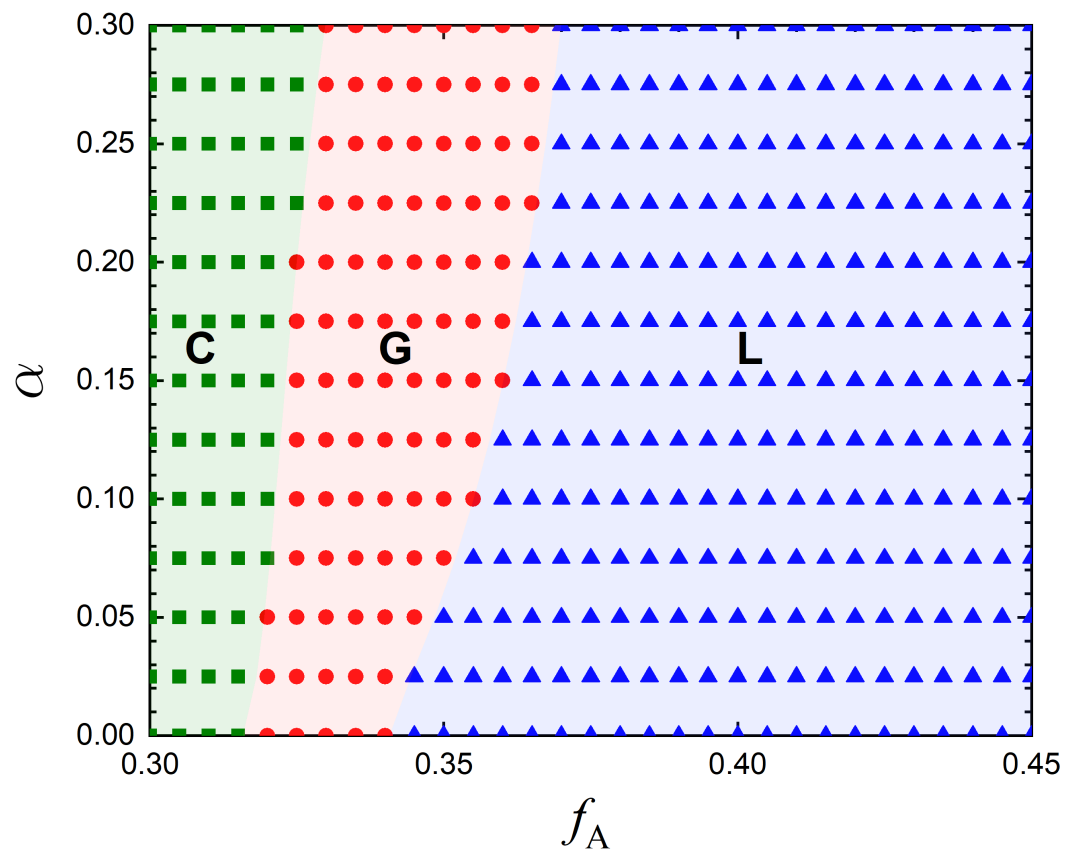

Figure S3: Phase diagram at fixed $\chi N_{\text {tot }}=36$ analogous to Figure $4 \mathrm{a}$ in the main text. Same symbols and labels are used as in Figure 4a. 


\section{Free energies of bottlebrush block polymer and coun- terpart diblock copolymer melts}
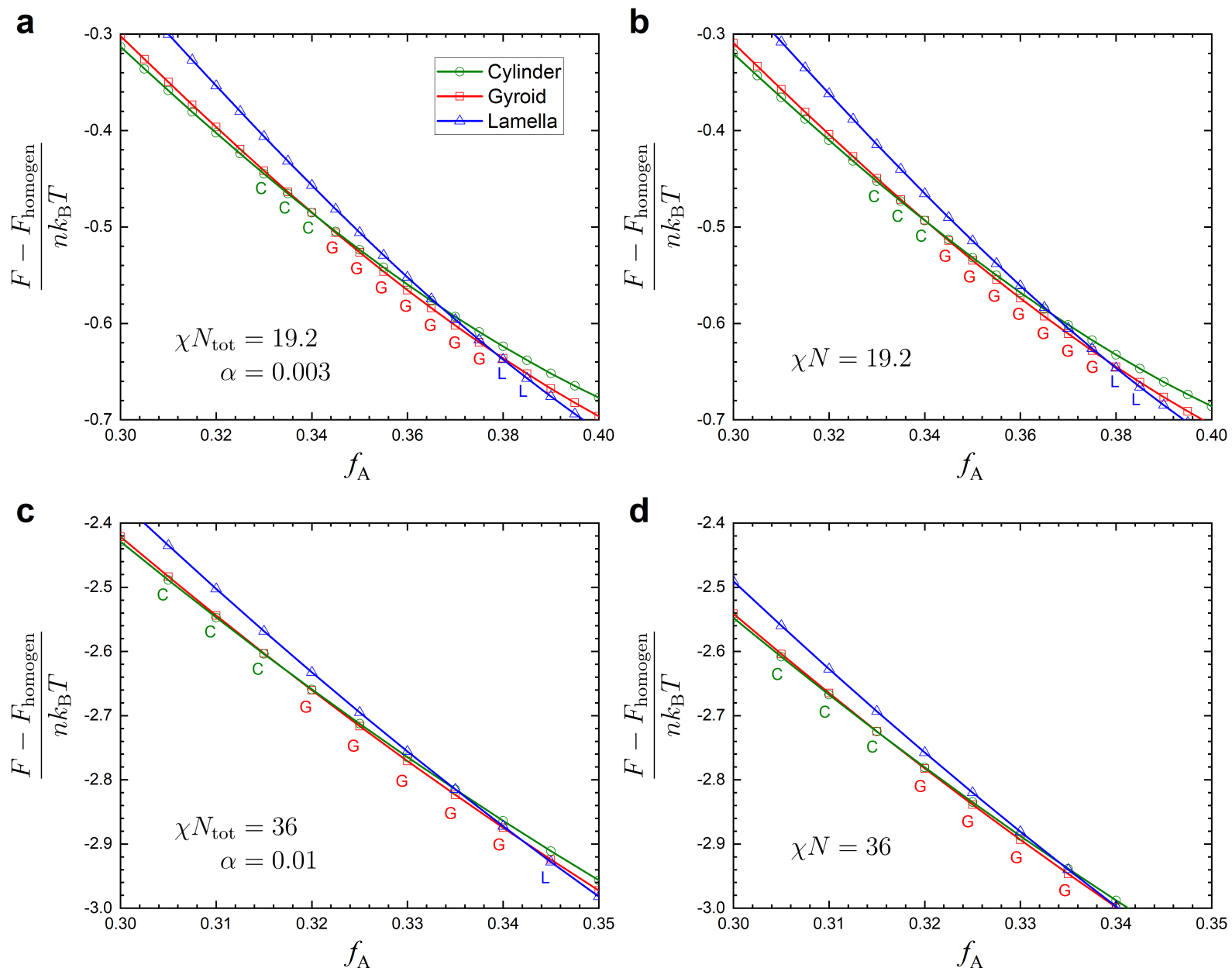

Figure S4: Relative free energies to the homogeneous disordered phase for the bottlebrush block polymers of $n_{\mathrm{SC}}=200$ at (a) $\alpha=0.003$ and (c) $\alpha=0.01$, and for their counterpart linear diblock copolymers at (b) $\chi N=19.2$ and (d) $\chi N=36$. 


\section{Lamellar periods of bottlebrush block polymer and counterpart diblock copolymer melts}

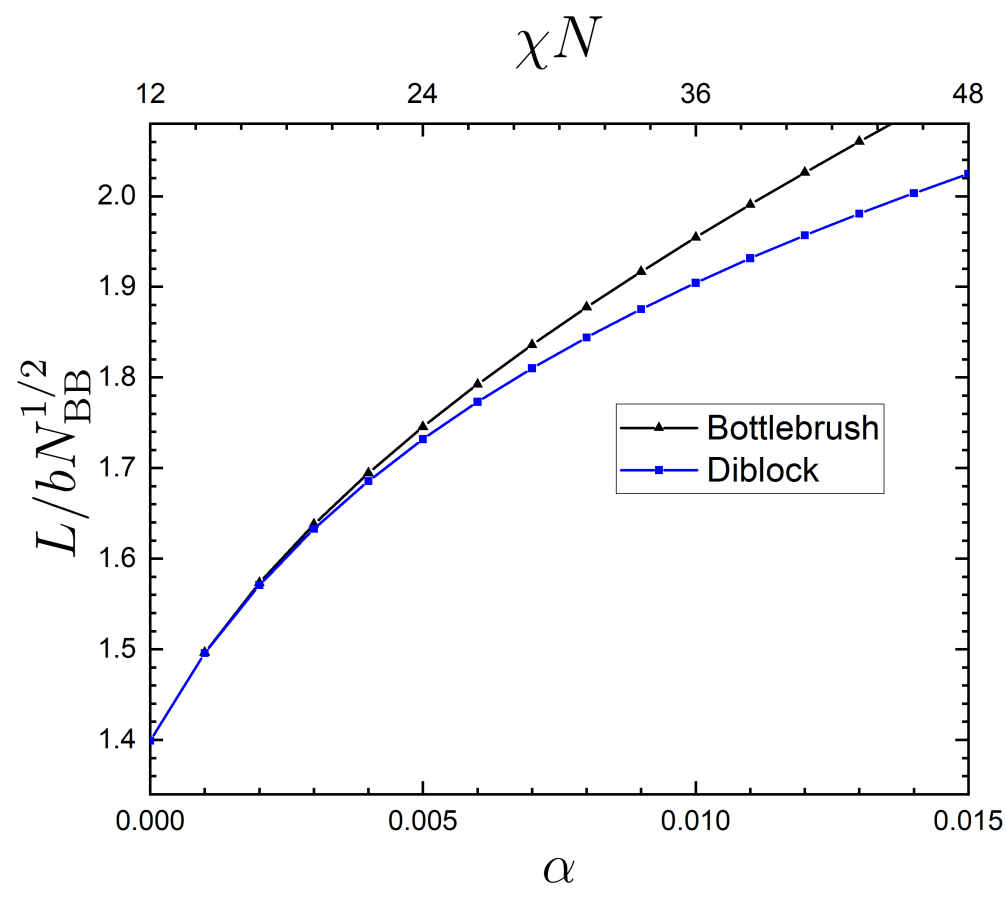

Figure S5: Lamellar periods (a) as a function of $\alpha$ for the compositionally symmetric $\left(f_{\mathrm{A}}=\right.$ $0.5)$ bottlbrush block copolymer melts at $\chi=12$ with $n_{\mathrm{SC}}=200$ and (b) as a function of equivalent $\chi N=\chi N_{\text {tot }}$ for the counterpart diblock copolymer melts.

\section{References}

(1) Ranjan, A.; Qin, J.; Morse, D. C. Linear response and stability of ordered phases of block copolymer melts. Macromolecules 2008, 41, 942-954.

(2) Arora, A.; Morse, D. C.; Bates, F. S.; Dorfman, K. D. Accelerating self-consistent field theory of block polymers in a variable unit cell. J. Chem. Phys. 2017, 146, 244902.

(3) Levi, A. E.; Lequieu, J.; Horne, J. D.; Bates, M. W.; Ren, J. M.; Delaney, K. T.; Fredrickson, G. H.; Bates, C. M. Miktoarm Stars via Grafting-Through Copolymerization: SelfAssembly and the Star-to-Bottlebrush Transition. Macromolecules 2019, 52, 1794-1802. 